\title{
Optimisation of Experimental Techniques to Enhance the Efficiency of
}

\section{Polymer Photovoltaic Cell}

\author{
P S Sodhi', Sanjay Sahare ${ }^{1}$, V. Naresh Kumar ${ }^{1}$, A.K. Swarnkar ${ }^{1}$, \\ S. R. Jadkar ${ }^{2}$, Balasubramanian K. ${ }^{3}$ and Tejashree Bhave ${ }^{1, *}$ \\ ${ }^{1}$ Deptartment of Applied Physics, DIAT (DU), Pune, India \\ ${ }^{2}$ School of Energy Studies, University of Pune, India \\ ${ }^{3}$ Deptartment of Material Engineering, DIAT (DU), Pune, India
}

‘Corresponding Author E-mail: tejashreebhave@diat.ac.in, tejab68@gmail.com

Keywords: Polymer Solar Cell, Device Fabrication and Characterization, MEHPPV, PCBM, PEDOT:PSS.

\begin{abstract}
The idea of generating electricity from plastics itself sounds enthralling, but it is equally challenging to achieve in the lab and then take it to the production level. Polymer Solar Cells (PSCs) have the advantage of lower cost and flexibility but they do suffer from problems like low efficiency and smaller service life which is mainly due to limited absorption spectra, poor charge mobility and the degradation of the polymers. A combination of narrow band donor and fullerene derivative is one of the possible approaches to fabricate a working device. In this work, various experimental techniques have been optimised in order to achieve better efficiency of the PSCs in atmospheric condition. Optimized parameters from polymer mixing ratio, spin coating, annealing and others were used to enhance efficiency of device. Parameters were optimized with the help various techniques viz. Viscometer, Thermo Gravity Analysis, UV-vis spectroscopy, Scanning Electron Microscopy and I-V measurement system. We report $0.76 \%$ efficiency in these solar cells, which were fabricated in open atmospheric condition. The as-prepared device showed a good performance with an opencircuit voltage $\left(\mathrm{V}_{\mathrm{oc}}\right) 0.74 \mathrm{~V}$, short circuit current $\left(\mathrm{I}_{\mathrm{sc}}\right) 0.6 \mathrm{~mA}$, a fill factor $(\mathrm{FF})$ of $35 \%$.
\end{abstract}




\section{Introduction}

In the present times when the energy requirements are escalating steeply and global warming is becoming cause of concern for the nations across the world, renewable energy sources and especially solar energy shows us the light at the end of the tunnel. Solar market currently is ruled by Silicon based technology which itself suffers from the disadvantage of high cost due to requirement of solar grade silicon [1,2]. In the recent times, conjugated polymers [3] have opened many windows of opportunities for the researchers and the industries by virtue of the advantage these polymers offer in terms of their conducting properties similar to that of semiconductors [4], flexibility, environment friendliness and of course the ease of fabrication [5-8]. All these features also assist in PSCs cost reduction [9] as compared to inorganic photovoltaic cell. Despite all these great advantages, PSCs suffer from several disadvantages such as low service life due to fast degradation and low efficiency [10-13].

In this work, fabrication of PSCs and improvements in $\mathrm{V}_{\mathrm{OC}}$ and $\mathrm{I}_{\mathrm{SC}}$ using a choice of characterisation facilities in open atmospheric condition was demonstrated. A cell with structure of ITO/PEDOT:PSS/MEHPPV+PCBM/Al was fabricated and each layer was formed after thorough optimisation and characterisation. A layer of Poly(3,4-ethylenedioxythiophene): poly(styrenesulfonic acid) (PEDOT:PSS) was deposited over Indium Tin Oxide (ITO) layer using spin coating technique, which effectively functions as hole conducting layer. A combination of narrow-band donor and fullerene derivative is a possible approach to active layer formation in the PSCs. Hence MEHPPV [poly 2-methoxy-5-(2'-ethyl-hexyloxy)-1,4-phenylene vinylene] and PCBM [phenyl-C61-butyric acid methyl ester], which is a fullerene derivative, acts as donor and acceptor respectively. This active layer could be coated by various techniques like spin coating, spray coating etc. After necessary surface treatment, the active layer was coated on aluminium by thermal evaporation technique. 


\section{Working of the Polymer Photovoltaic cell}

Difference in Inorganic and Organic photovoltaic cells. Whenever the inorganic solar cells are in discussion, exciton generation and separation of electron and hole is never a matter of crisis but in organic solar cells this issue needs a very careful handling. In case of Silicon solar cell, if we try to find the binding energy and exciton radius by the formula-

Binding Energy, $\mathrm{E}_{\mathrm{b}}=13.6 \times 1 / \mathrm{K}^{2} \mathrm{x}\left(\mathrm{m} / \mathrm{m}_{0}\right) \mathrm{eV}, \mathrm{K}$ being the dielectric constant

Radius of exciton, $\mathrm{r}_{\mathrm{b}}=0.53 \times \mathrm{K} \times\left(\mathrm{m}_{0} / \mathrm{m}\right) \AA$

For $\mathrm{Si}$, substituting, $\mathrm{K} \sim 12$ and $\mathrm{m}=0.1 \mathrm{~m}_{0}$, we get the binding energy of $13.6 \mathrm{meV}$ and radius as 53 $\AA$. And the value of $\mathrm{kT}(\mathrm{k}$ being boltzman constant and $\mathrm{T}$ is the temperature $)$ at room temperature is $25 \mathrm{meV}$. Now if we look into the process of electron hole pair generation in silicon solar cell, a photon is being absorbed and an exciton is being formed with the radius of $53 \AA$. Since the binding energy is of the order of $13.6 \mathrm{meV}$, a lattice vibration at room temperature which has the energy of $25 \mathrm{meV}$ is sufficient to separate electron. If we apply similar treatment to a polymer in which the value of dielectric constant is $\mathrm{K} \sim 3$ which is very low. Hence we get $\mathrm{E}_{\mathrm{b}}$ of the order of $151 \mathrm{meV}$ and $\mathrm{r}_{\mathrm{b}}$ as $15 \AA$. Therefore, we atleast need 6 kT's to separate exciton in PSCs and at room temperatures they recombine after a while and there is no energy coming out. To overcome this problem, in place of a single polymer, we have mixed two different polymers with different work functions. This is leading to formation of sharp junction and it may be sharper than $15 \AA$ which consists of approximately 6-7 atoms. When an exciton comes in the vicinity of such a junction, this sharp field will pull out electron and hole apart, so the need of hetero materials in PSCs is inevitable. Further to avoid recombination at the defective interfaces, two different materials Aluminium and ITO with different work functions are used to pull the electron and holes to the respective electrodes. Ripping apart the exciton is the role of heterojunction and sweeping of electron and hole is carried by built in field. The process is depicted in Fig.1. 


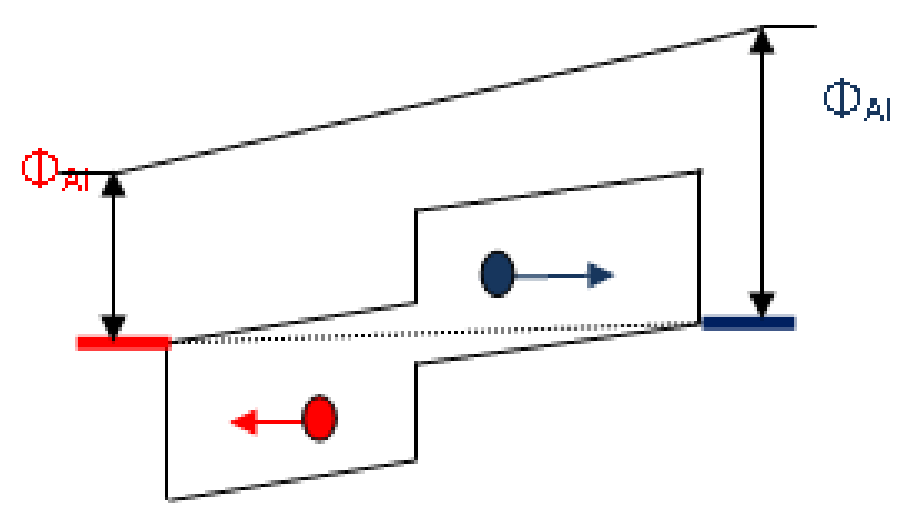

Fig. 1. Sharp Junction created due to hetero junction polymers and use of two electrodes with different work functions.

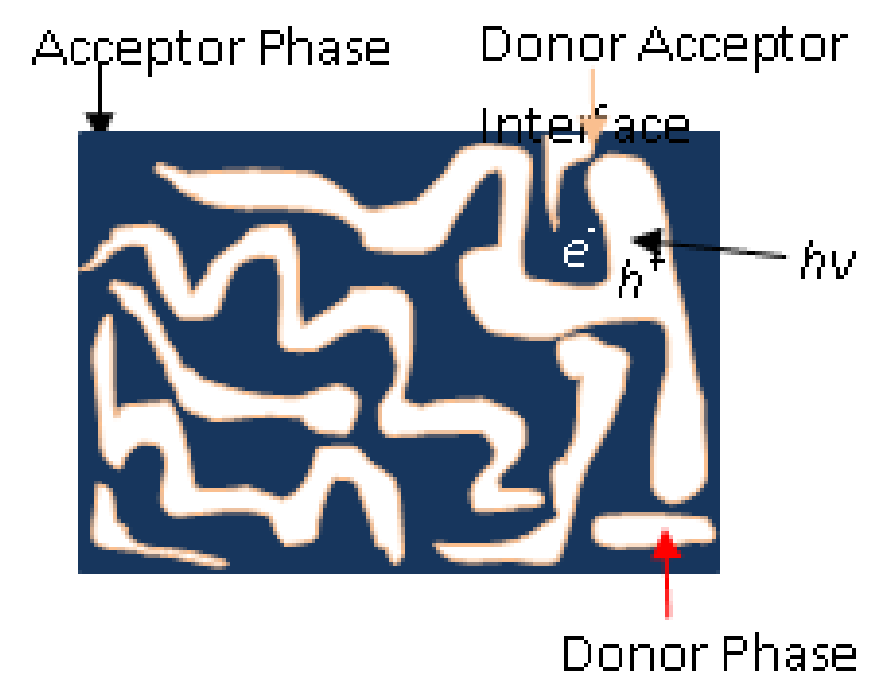

Fig. 2. Phase separation between two polymers due to no entropy of mixing known as spinodal decomposition.

If polymer A and Polymer B are mixed with some organic solvent, they get phase separated as there is no entropy of mixing unlike the case of salt in water. In case of high molecular weight polymers, as the polymerisation index gets large, the entropy of mixing approaches zero. The process of phase segregation after mixing of polymers is better known as spinodal decomposition as depicted in Fig. 2. 
General Principle of Working. In this section, the steps involved in the working of a PSCs are elucidated. Broadly, these can be classified into- (i) Excitons formation due to absorption of light (Photons), (ii) Separation of exciton into electron and hole and (iii) Collection of electron and hole

Exciton Formation due to Absorption of Photons. Incident photon can cause either absorption or scattering, in case of absorption if the energy is more than a minimum value; the electron gets promoted to conduction band leaving a hole in the valence band. This electron hole pair forms the excitons. If we consider case of PSC, electron gets promoted from Valence band i.e. Highest Occupied Molecular Orbit (HOMO) to conduction band i.e. Lowest Unoccupied Molecular Orbit (LUMO) [2]. The complete process effectively states the photovoltaic effect and is shown in 4 steps in Fig. 3.
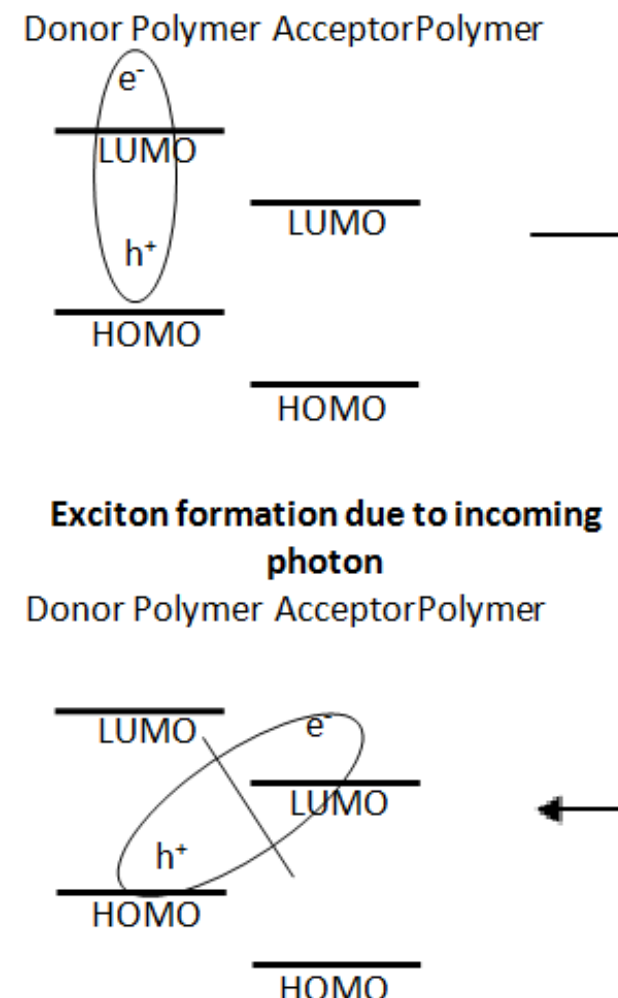

Electron hole pair formed due to sharp junction
Donor Polymer AcceptorPolymer

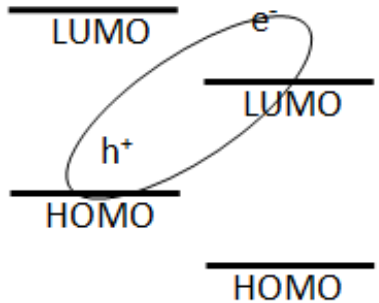

Charge transfer from donor to acceptor

Donor Polymer AcceptorPolymer

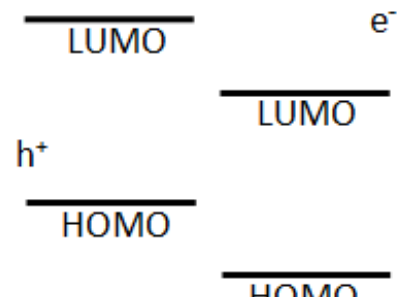

Charge collection at respective electrodes

Fig. 3. Process of electron hole pair generation and charge separation demonstrated in 4 steps. 
Separation of Exciton into Electron and Hole. As discussed before, due to low dielectric constant of the polymers, it becomes difficult to break the exciton into electron hole pair and hetero materials are used in order to create a sharp junction which like a knife gets penetrated into exciton coming in the junction region, hence separating electron from the hole. Also since the diffusion length of electron hole pair is very less, Bulk Hetero Junction (BHJ) concept is being used, wherein a blend of two polymers is formed after mixing in some kind of organic solvent.

Collection of Electron and Hole. When the blend of two polymers is formed, it acts like a bicontinuous interpenetrating network. This bicontinuous interpenetrating network functions as the collection mechanism for the electrons and holes. As seen in the Fig. 4, gray circles are the donor polymer and the black lines represents fullerene, they can be imagined to be fully inter percolated. So if electron is transferred from donor polymer to the acceptor, it can find its path to the electrode. For this, 10-20 nm link scale is required to avoid losing electron hole pair before they reach their respective electrodes.

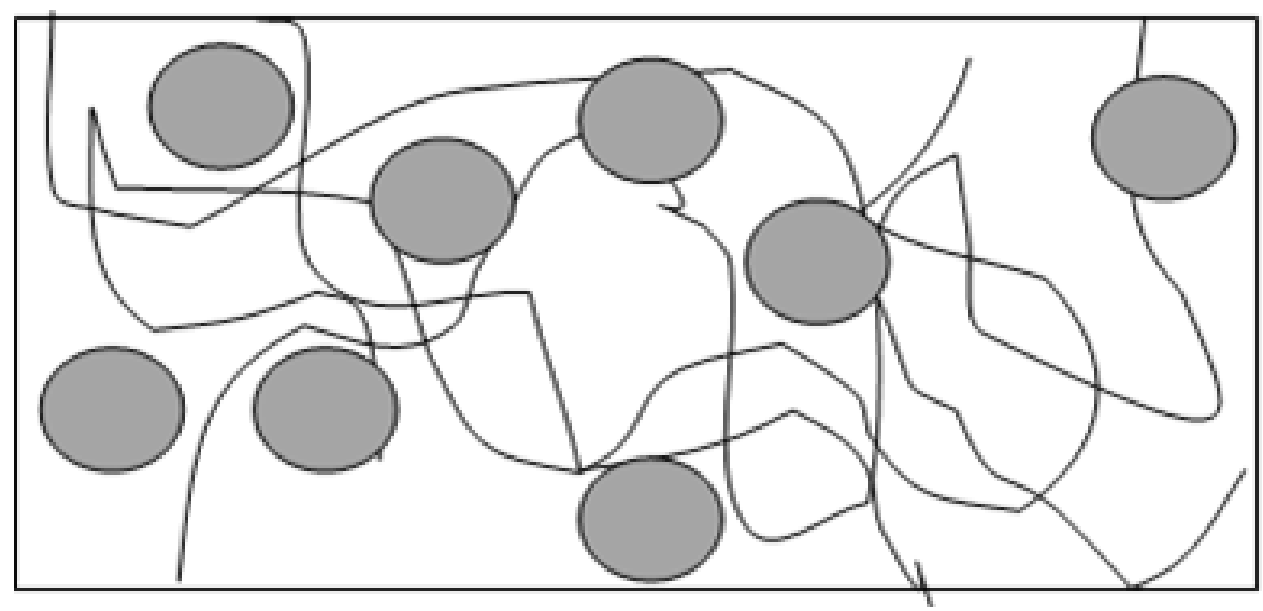

Fig. 4. Bicontinuous interpenetrating network functions as the collection mechanism for the electrons and holes. 


\section{Experimental}

Procurement of Material and Preparation of Sample. All the chemicals and substrate required for fabrication of PSCs were procured from Sigma Aldrich. The surface resistivity of procured ITO coated glass was 70-100 $\Omega-\mathrm{cm}^{-2}$. PEDOT:PSS [Poly (2, 3-dihydrothieno-1, 4-dioxin)-poly (styrenesulfonate)] was purified with $0.22 \mu \mathrm{m}$ PTFE [Polytetrafluoroethylene] filter. HPLC grade ODichloro benzene $\left[\mathrm{C}_{6} \mathrm{H}_{4} \mathrm{Cl}_{2}\right]$ was used as a solvent for the active layer. The solution for active layer was prepared with MEH-PPV: PCBM [Poly [2-methoxy-5-(2-ethylhexyloxy)-1, 4phenylenevinylene]: PCBM [phenyl-C61-butyric acid methyl ester] weight ratio of 1:4 and the solvent was added with a scale of $18 \mathrm{mg} / \mathrm{ml}$ [14]. The solution was put to stirring for a period of 12 hours before coating.

Coating Technique. For deposition of the PEDOT:PSS and active layer, two different techniques were explored, spin coating and spray coating. Spray coating involves forcing the printing ink through a nozzle whereby a fine layer was formed, whereas spin coating involves application of a liquid to a substrate followed by acceleration of the substrate to a chosen rotational speed. Spin coating is advantageous over spray coating in terms of reproducing thickness and morphology control. At the same time spray coating is beneficial when it comes to reduction in wastage of polymer solution. Both the techniques were tried, since control over the thickness of the film is a primary criterion for fabricating a successful device, spin coating technique was optimised for further experiments towards coating of active layer [3].

Fabrication of the Device. The stack with different layers of fabricated cell is shown in Fig. 5. The ITO coated glass substrate was subjected to various steps of cleaning. The substrate was thoroughly rinsed in ultra sonic bath with the lab detergent followed by cleaning with distilled water. Cleaned substrate was put again for sonication in acetone at a temperature of $50{ }^{\circ} \mathrm{C}$ followed by thorough cleaning in isopropyl alcohol. The substrate was dried in the Oven for 15 minutes at $100{ }^{\circ} \mathrm{C}$. Among 
the various available coating techniques, spin coating technique was used for depositing different layers of the PSC. $200 \mu 1$ of PEDOT: PSS solution was used to deposite the first layer over the substarte by spin coating technique. The coating speed was $1500 \mathrm{rpm}$ for a period of 60 seconds. This was followed by thermal annealing at a temperature of $120{ }^{\circ} \mathrm{C}$ for 10 minutes. After the layer drying, the substrate was coated with the active layer solution. The parameters for spinning the active layer solution were optimised from $750 \mathrm{rpm}$ to $1500 \mathrm{rpm}$ for a period of 60 seconds. We got best results on the basis of unifrormity and thickness of the film with $750 \mathrm{rpm}$. Hence we continued furhter experiments with that parameter. The substrate was again subjected to thorough annealing at a temperature of $120^{\circ} \mathrm{C}$ for 30 minutes. Second electrode was deposited by vacuum evaporation technique. The aluminium electrode was deposited on the active layer at pressure of $1 \times 10^{-6}$ Torr. The solar cell was then taken out of the vacuum chamber and subjected to testing in IV measurement system.

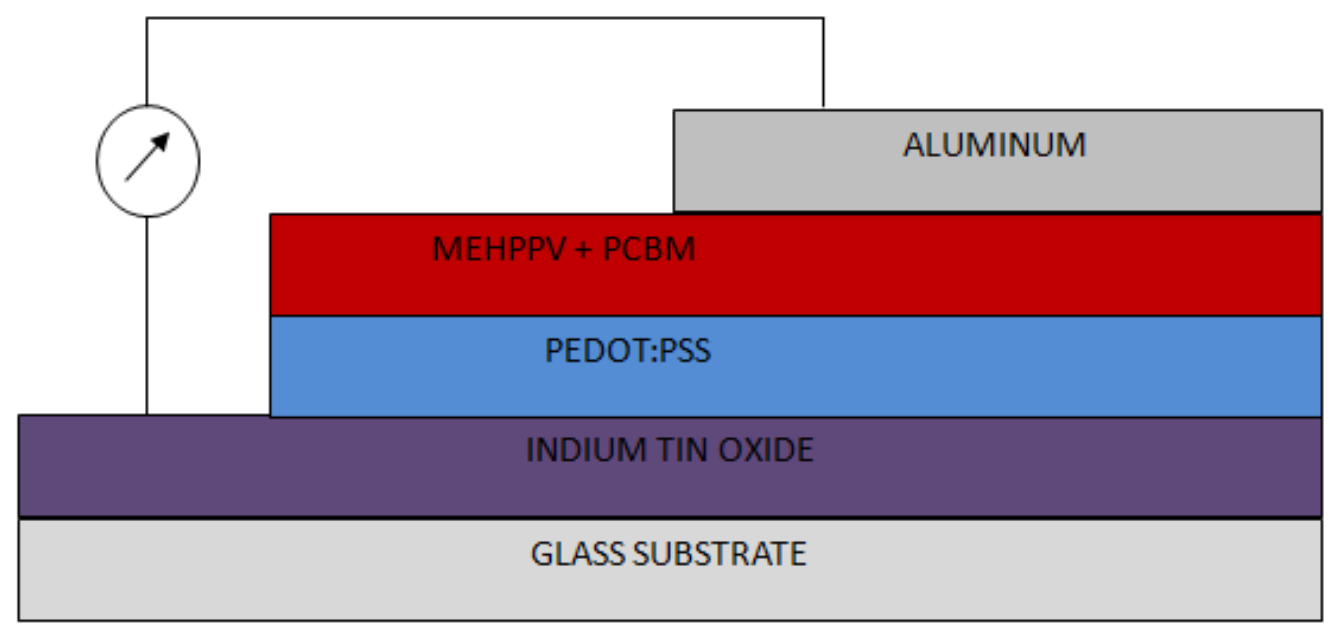

Fig. 5. Structure of the fabricated polymer solar cell. 
Characterization Techniques. The absorption spectra of the dye were recorded using UV-Vis spectrophotometer (Ocean Optics). The viscosity measurement of the prepared blend solution was tested using the Brookfield Viscometer. Morphology of the film was checked by Scanning Electron Microscope (JEOL) at $5 \mathrm{KV}$. Degradation of the material were identified with the help Thermogravimetry analysis (TGA) from Perkin Elmer STA 6000. The IV Measurement System consisted of Newport solar simulator (AM 1.5 standards) and Keithley source meter 2420.

\section{Results and Discussion}

UV-Visible Spectroscopy. UV Visible absorbance at differnt layers of the stack shown in Fig. 6. It confirms that active layer which is combination of MEHPPV and PCBM absorbs light effectively between 350 to $700 \mathrm{~nm}$ with few peaks around $500 \mathrm{~nm}$. As expected, ITO and glass does not absorb much of the light in the visible region and even not in the area where MEHPPV:PCBM absorbs the light. When PEDOT: PSS layer is applied, a very low absorbance was observed. This low and negative kind of absorbance has an effect of reducing the reflective ability of the glass, as a result more light is getting transmitted to the active layer.

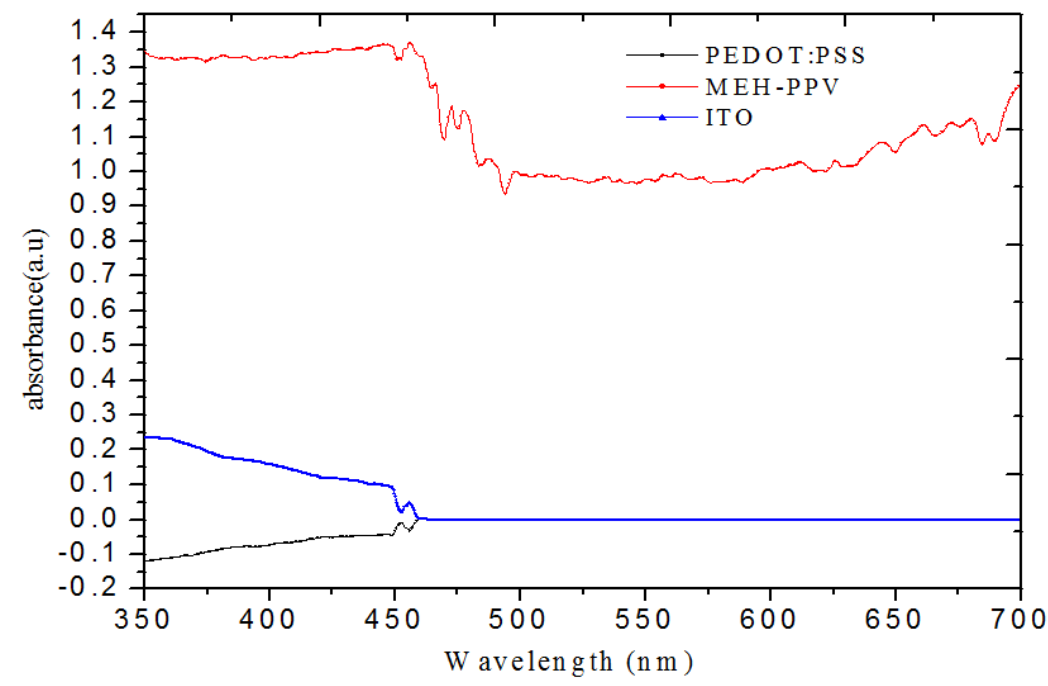

Fig. 6. Absorption spectra of various layers of the stack. 
Simultaneous Thermal Analysis (STA). The degradation mechanism in PSC depends upon the intensity of the incident light and annealing of the material. Solar cell has to be exposed to the incident sunlight to create charge carriers. As discussed in theory, in order to achieve spinodal decomposition, thermal annealing plays an important role. TGA/DSC of the MEHPPV and PCBM revealed that they reduce to their weight percentage of $95 \%$ at the temperatures $150{ }^{\circ} \mathrm{C}$ and $205{ }^{\circ} \mathrm{C}$ respectively as shown in Fig. 7. Based on this data, the annealing temperature during the experiment was optimised to $120^{\circ} \mathrm{C}$.

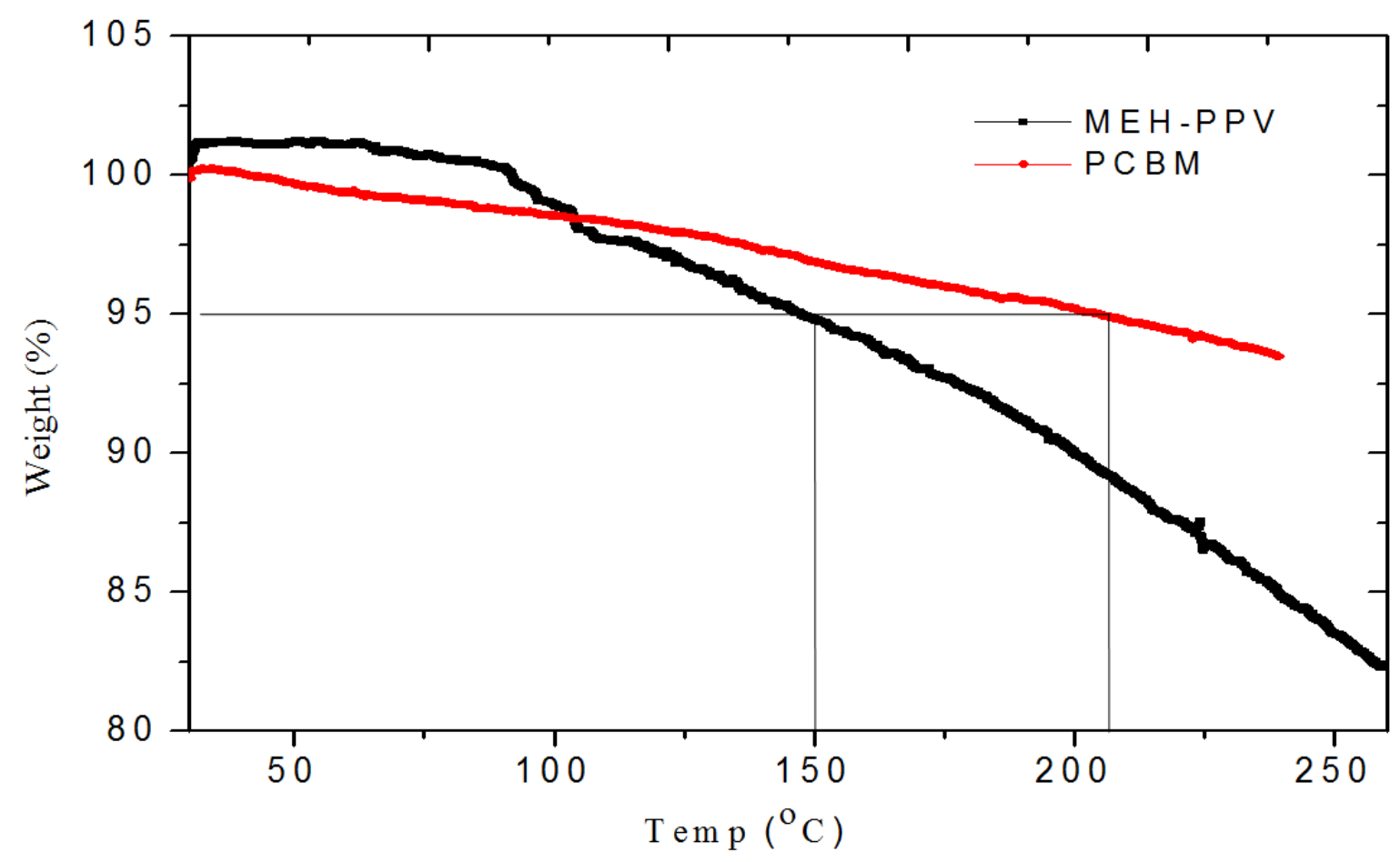

Fig. 7. Simultaneous Thermal Analysis (STA) of polymers to assess suitable temperature for annealing to avoid degradation.

Rheological Measurements.Viscosity is a measure in terms of resistance of a fluid which is being deformed by shear stress. Put simply, the less viscous the fluid is, the greater its ease of movement (fluidity). This measurement will be helpful especially when the solar cells are in production. If PSCs are printed with ink jet technology, the period for which the solution of polymers is transferred from Tank to the thin film, the viscosity should not change beyond limit. In case due to sheer stresses 
the viscosity changes, it will result in change in the thickness of the active layer, which can be deteriorate the output of the solar cell. The change was observed for a period of 1 minute at different stress rates. The graph between viscosity and sheer rate is shown in Fig. 8 .

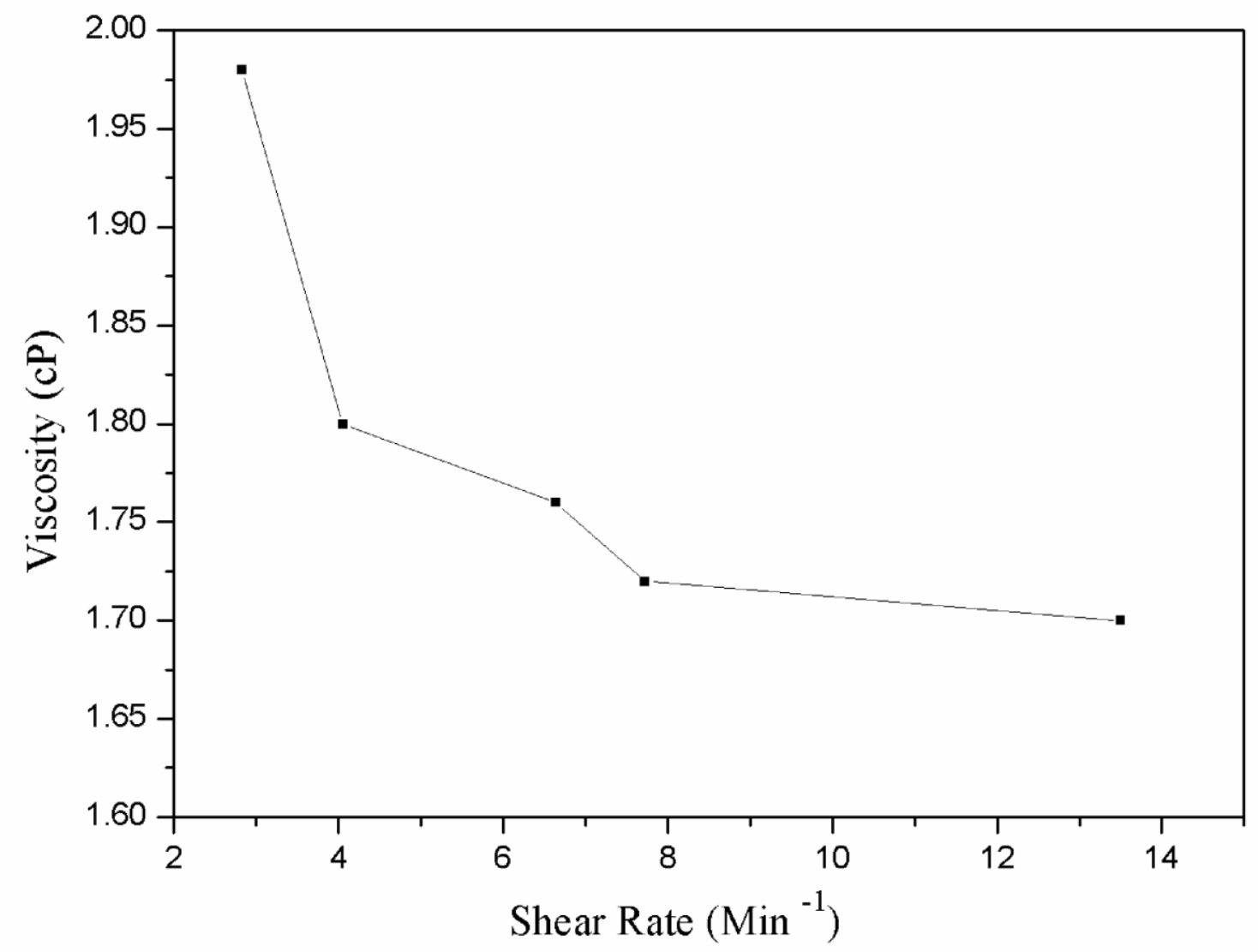

Fig. 8. Rheological measurements at different shear rates to confirm any change in viscosity of the fluid. 


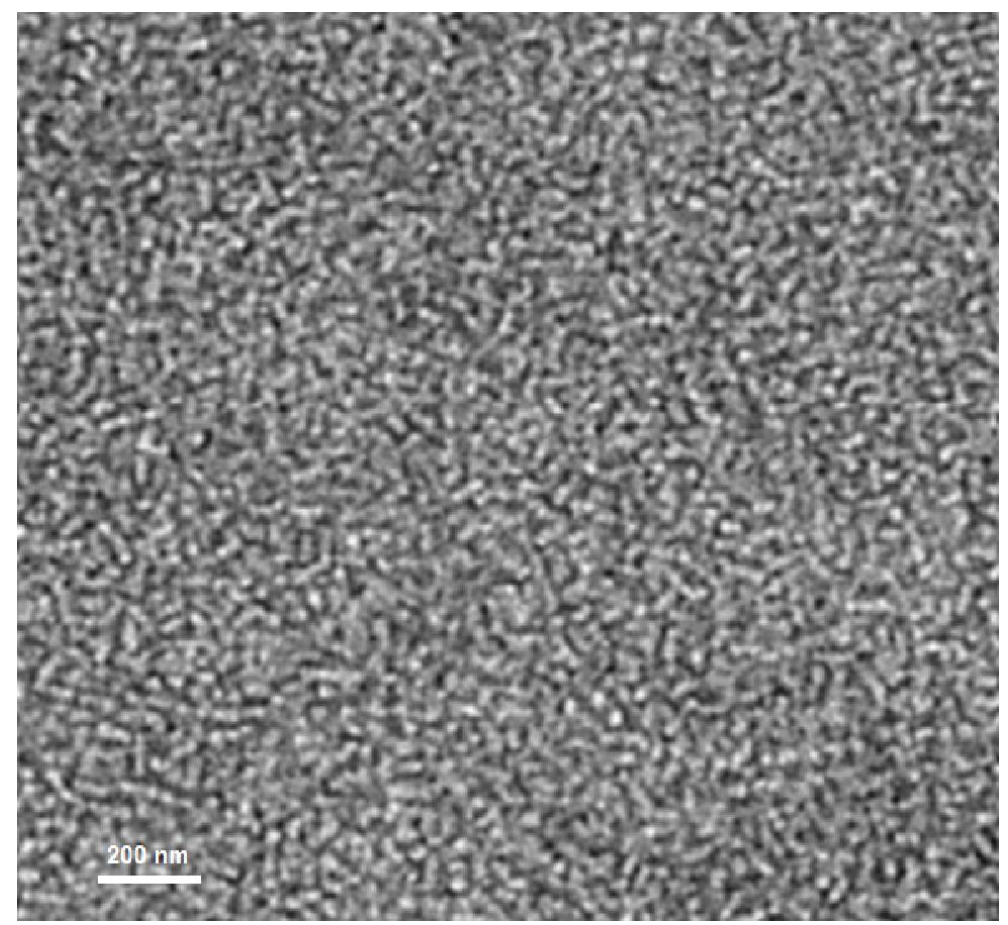

Fig. 9. Scanning Electron Microscopy structure of Active layer.

Scanning Electron Microscopy. The active layer of the cell was subjected to Scanning Electron microscopy (SEM) after annealing for 10 minutes at a temperature of $120^{\circ} \mathrm{C}$, which is shown in following Fig. 9. In this image a quasi periodic structure can be observed. The contrast seen in the image is because of the cluster of carbon atoms in PCBM. Though there will be carbon atoms in polymer, but large cluster of carbon atoms in the PCBM is responsible for this contrast. The phase separation introduced between two polymers can be clearly made out, which is also called as spinodal decomposition.

IV Measurement. The I- $V$ characteristics of the as-prepared devices under AM 1.5 illumination with an intensity of $100 \mathrm{~mW} / \mathrm{cm}^{2}$ are shown in Fig. 10. The recorded parameters of the solar cell are tabulated in Table 1. The as-prepared device showed a good performance with an open-circuit voltage $\left(\mathrm{V}_{\mathrm{oc}}\right) 0.74 \mathrm{~V}$, short circuit current $\left(\mathrm{I}_{\mathrm{sc}}\right) 0.6 \mathrm{~mA}$, a fill factor $(\mathrm{FF}) 35 \%$, and efficiency of 0.76 $\%$. A point here to note is that the ITO sheet used had a resistivity of the order of $70-100 \Omega \mathrm{cm}^{-2}$ and the complete process was carried out in an open atmosphere. Researchers have achieved efficiency 
of more than $2.5 \%$ in the closed atmosphere. While this experiment was earlier performed with toluene as solvent and spray coating technique, a very low efficiency of the order of $0.008 \%$ was achieved in the open atmosphere. Organic solvent plays an important role and solubility in non chlorinated solvents such as toluene or xylene is not good as chlorinated based solvents [4]. Comparison between parameters of cells is shown in Table 1. Therefore, changes in the coating technique and organic solvent helps to improve the packing density of the film which leads to increase the efficiency up to $0.76 \%$ in the open atmospheric condition. IV Curve is shown in Fig. 10. Values of $\mathrm{I}_{\mathrm{SC}}, \mathrm{V}_{\mathrm{OC}}, \mathrm{FF}$ and PCE employed in this study as measured on samples under ambient atmosphere conditions, AM 1.5 spectral distributions and $100 \mathrm{~mW}-\mathrm{cm}^{-2}$.

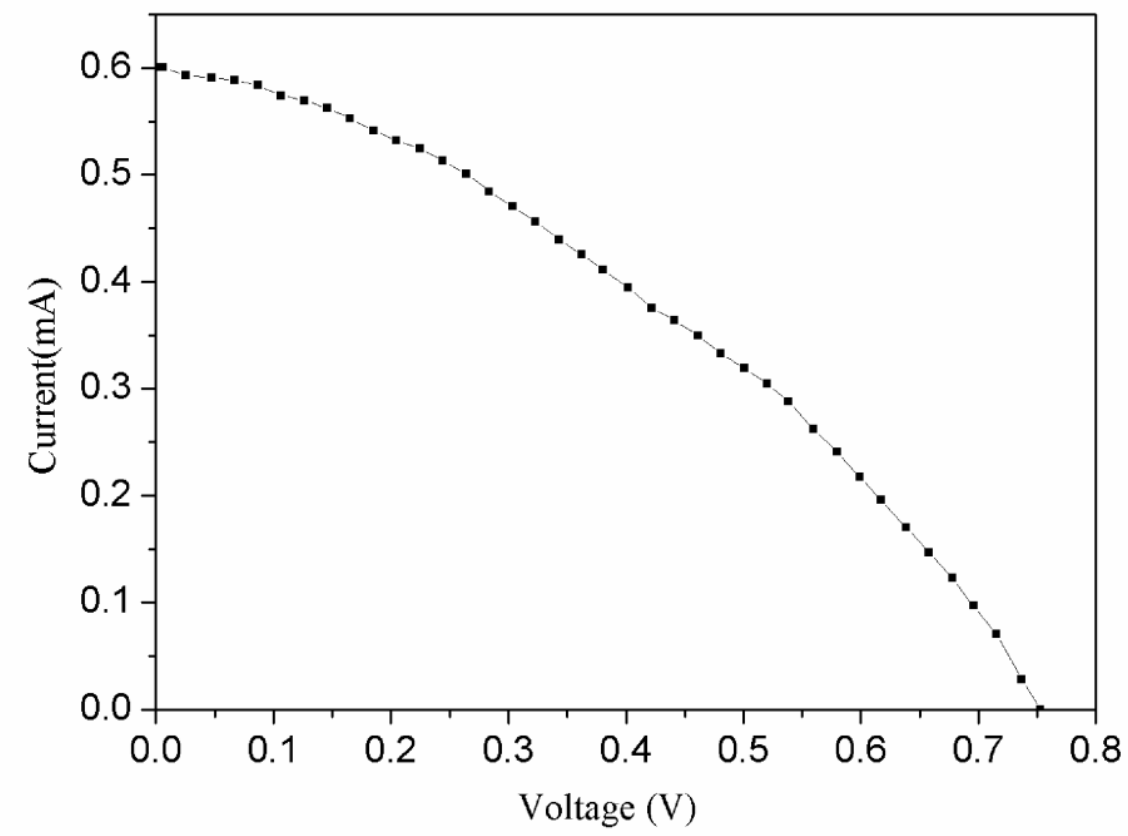

Fig. 10. IV curve of the fabricated cell under the standard test condition AM 1.5 
Table 1. Recorded parameters of the fabricated solar cell.

\begin{tabular}{|c|c|c|c|c|c|c|}
\hline $\begin{array}{l}\text { Cell } \\
\text { No. }\end{array}$ & $\begin{array}{c}\text { Device Type, Geometry and Coating } \\
\text { technique }\end{array}$ & $\begin{array}{c}\text { Isc } \\
(\mathbf{m A})\end{array}$ & $\begin{array}{c}\mathrm{J}_{\mathrm{SC}} \\
\left(\mathrm{mA}-\mathrm{cm}^{-2}\right)\end{array}$ & $\begin{array}{l}\mathbf{V}_{\text {OC }} \\
(\mathrm{V})\end{array}$ & FF (\%) & $\begin{array}{l}\text { PCE } \\
(\%)\end{array}$ \\
\hline 1 & $\begin{array}{c}\text { ITO/PEDOT:PSS/MEHPPV+PCBM+D } \\
\text { ichloroBenzene/Al } \\
\text { (Spin Coating Technique) }\end{array}$ & 0.6 & 3.306 & 0.740 & 35.67 & 0.758 \\
\hline 2 & $\begin{array}{l}\text { ITO/PEDOT:PSS/MEHPPV+PCBM+T } \\
\text { oluene/Al (Spray Coating Technique) }\end{array}$ & 0.031 & 0.031 & 0.704 & 16.7 & 0.008 \\
\hline
\end{tabular}

\section{Summary}

In this paper, we have demonstrated a straightforward approach to fabricate a PSCs with decent photo conversion efficiency in an open atmospheric condition. To achieve better efficiency, we optimised all the nesessary parameters and carried out the fabrication of PSCs using the best recipe. Also the improvement was observed when we brought a change in the coating technique and organic solvent. Rearrangement of polymers by spinodal decomposition was also observed well brought out in this paper. With the open atmosphere process it is very easy to prepare PSCs for large scale applications. Further improvement in efficiency is still required for commercial viability of PSCs.

\section{Acknowledgement}

The authors would like to thank Vice-Chancellor of DIAT, Pune for extending all the facilities of the institute. Tejashree Bhave and Sanjay Sahare acknowledge financial support from DRDO-DIAT Program on Nano Materials from ER-IPR of Defence Research and Development Organization. 


\section{References}

[1] D.E. Carlson,C.R. Wronski, Amorphous silicon solar cell, App. Phy. Lett. 28 (1976) 671-673.

[2] K. Yamamoto, M. Yoshimi, Y. Tawada, Y. Okamoto, A. Nakajima, Cost effective and high performance thin film Si solar cell towards the 21st century, Sol. Energy Mater. Sol. Cells 66 (2001) 117-125.

[3] Tingbin Yang and et.al, Inverted polymer solar cells with $8.4 \%$ efficiency by conjugated polyelectrolyte, Energy Environ. Sci. 5 (2012) 8208-8214.

[4] Y. Liang and et.al, Development of New Semiconducting Polymers for High Performance Solar Cells, J. Am. Chem. Soc. 131 (2009) 56-57.

[5] C.J. Brabec, Organic photovoltaics: technology and market, Sol. Energy Mater. Sol. Cells 83 (2004) 273.

[6] G. Li, L. Liu, F. Wei, S. Xia, X. Qian, Recent Progress in Modeling, Simulation, and Optimization of Polymer Solar Cells, IEEE J. Photovoltaics 2(3) (2012) 320-340.

[7] F.C. Krebs, Fabrication and processing of polymer solar cells: A review of printing and coating techniques, Sol. Energy Mater. Sol. Cells 93 (2009) 394-412.

[8] M.N. Yusli, T. Way Yun, K. Sulaiman, Solvent effect on the thin film formation of polymeric solar cells, Mater. Lett. 63 (2009) 2691-2694.

[9] D.M. Tanenbaum, H.F. Dam, R. Rösch, M. Jørgensen, H. Hoppe, F.C. Krebs, Edge sealing for low cost stability enhancement of roll-to-roll processed flexible polymer solar cell modules, Sol. Energy Mater. Sol. Cells 97(2012) 157-163.

[10] M. Jorgensen, K. Norrman, F.C. Krebs, Stability/degradation of polymer solar cells, Sol. Energy Mater. Sol. Cells 92 (2008) 686-714. 
[11] H.Yu Chen, J. Hou, S. Zhang, Y. Liang, G.Yang, Y. Yang, L. Yu, Y. Wu, Gang Li, PSCs with enhanced open-circuit voltage and efficiency, Nat. Photonics 3 (2009) 649-653.

[12] F.C. Krebs, H. Spanggaard, Significant Improvement of Polymer Solar Cell Stability, Chem. Mater. 17 (2005) 5235-5237.

[13] G. Shao, G.E. Rayermann, E.M. Smith, D.S. Ginger, Morphology-Dependent Trap Formation in Bulk Heterojunction Photodiodes, J. Phys. Chem. B 117(16) (2013) 4654-4660.

[14] F.C. Krebs, J.E. Carlé, N. Cruys-Bagger, M. Andersen, M.R. Lilliedal, M.A. Hammond, S. Hvidt, Lifetimes of organic photovoltaics: photochemistry, atmosphere effects and barrier layers in ITO-MEHPPV: PCBM-aluminium devices, Sol. Energy Mater. Sol. Cells 86 (2005) 499-516. 